Revista de

CIENCIAS AMBIENTALES

Tropical Journal of Environmental Sciences

\author{
BIOGRAFÍA
}

\title{
Douglas Robinson: herpetólogo, mentor y conservacionista
}

\author{
Douglas Robinson: Herpetologist, Mentor and Conservationist
}

\section{Anny Chaves Quirós ${ }^{a}$}

a Bióloga, conservacionista y consultora independiente. Heredia, Costa Rica, annychaves@ice.co.cr

Director y Editor:

Dr. Sergio A. Molina-Murillo

Consejo Editorial:

Dra. Mónica Araya, Costa Rica Limpia, Costa Rica Dr. Gerardo Ávalos-Rodríguez. SFS y UCR, USA y Costa Rica Dr. Olman Murillo Gamboa, ITCR, Costa Rica

Dr. Luko Hilje, CATIE, Costa Rica

Dr. Arturo Sánchez Azofeifa. Universidad de Alberta-Canadá

Asistente:

Joseline Jimenez Brenes

Editorial:

Editorial de la Universidad Nacional de Costa Rica (EUNA)
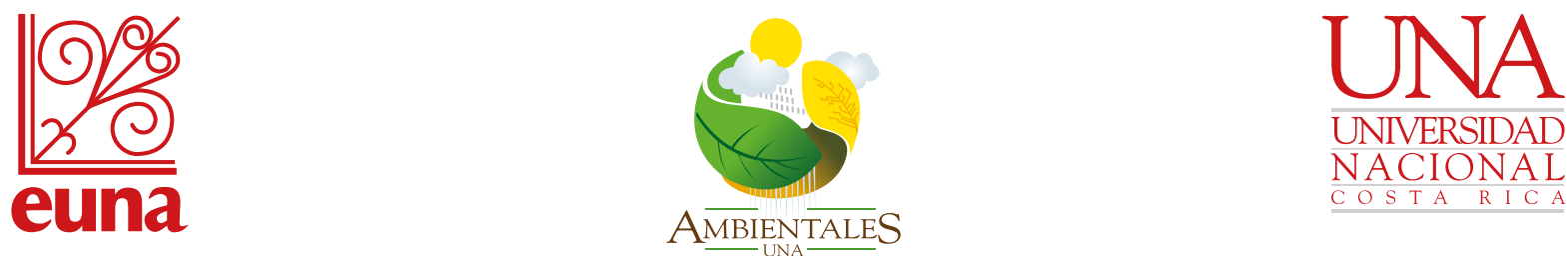

Los artículos publicados se distribuyen bajo una licencia Creative Commons Reconocimiento al autor-No comercial-Compartir igual 4.0 Internacional (CC BY NC SA 4.0 Internacional) basada en una obra en http://www.revistas.una.ac.cr/ambientales, lo que implica la posibilidad de que los lectores puedan de forma gratuita descargar, almacenar, copiar y distribuir la versión final aprobada y publicada (post print) del artículo, siempre y cuando se realice sin fines comerciales, no se generen obras derivadas y se mencione la fuente y autoría de la obra. 


\title{
BIOGRAFÍA
}

\section{Douglas Robinson: herpetólogo, mentor y conservacionista}

\author{
Douglas Robinson: Herpetologist, Mentor and Conservationist
}

\author{
Anny Chaves Quirós ${ }^{a}$
}

\section{Introducción}

Los anfibios y reptiles de Costa Rica han contado con el privilegio de capturar el interés de cientos de investigadores que desfilan por los diferentes ecosistemas del país, para realizar recolecciones, observaciones de comportamiento y documentar la maravillosa diversidad que este gran conjunto encierra. Los primeros naturalistas que llegaron a Costa Rica con diversos intereses de estudio no dejaron de caer en el encanto y desde mediados del siglo XIX muchas de las especies aportadas a la ciencia se documentaron con especímenes provenientes de Costa Rica.

En su libro Herpetofauna de Costa Rica, Savage (2002) detalla los aportes de pioneros de la herpetología de Costa Rica, entre los que se encuentran Emmett R. Dunn, Edward H. Taylor, Archie Carr y varios más. Si bien se llegó a contar con muy buena información de la herpetofauna, la prueba documental se encontraba en museos fuera del país, y el material de anfibios y reptiles que había sido depositado en el Museo
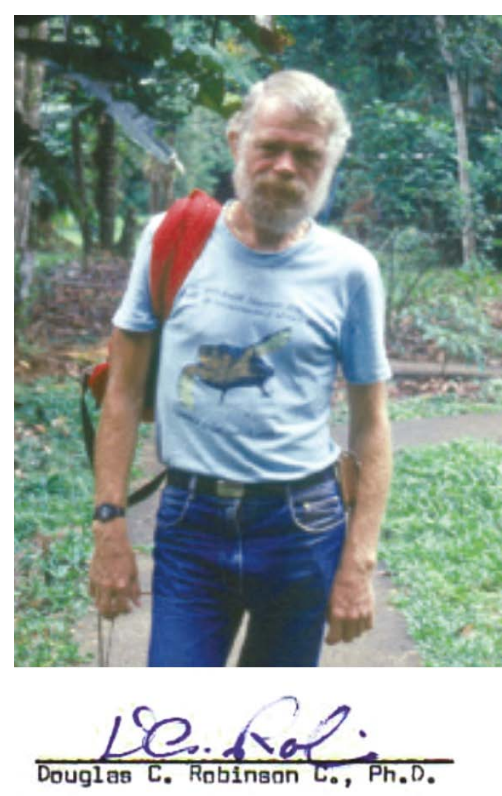
Nacional de Costa Rica era poco y en mal estado, debido a que no había un curador específico.

En el año de 1966 llegó a Costa Rica un herpetólogo cuyo nombre completo era Douglas Robinson Clark. Lo hizo como profesor invitado del Departamento de Biología de la Universidad de Costa Rica (UCR) cuando todavía era estudiante de doctorado en la Universidad de Texas A\&M.

Fue Douglas quien identificó esa necesidad de contar con la documentación física para la herpetofauna de Costa Rica y se propuso la meta de constituir la colección de anfibios y reptiles de nuestro país, misión que trascendió en la fundación del Museo de Zoología de la Universidad de Costa Rica. Douglas se convirtió en el eje del conocimiento de la herpetofauna nacional, tuvo la habilidad de aglutinar y colaborar con los estudiosos que nos visitaban, de los anfibios

a Bióloga, conservacionista y consultora independiente. Heredia, Costa Rica, annychaves@ice.co.cr

\begin{tabular}{|c|c|c|}
\hline 208 & $\begin{array}{l}\text { Creative Commons (Reconocimiento al autor-No comercial-Compartir igual } 4.0 \text { Internacional } \\
\text { (CC BY NC SA } 4.0 \text { Internacional) }\end{array}$ & $\begin{array}{l}\text { Revista de } \\
\text { CIENCIAS AMBIENTALES } \\
\text { Tropical Journal of Environmental Sciences }\end{array}$ \\
\hline
\end{tabular}


y reptiles, de tal forma que la información no se fugara. Organizó expediciones a sitios poco conocidos y participó junto con sus estudiantes en campañas de recolección para enriquecer las colecciones del recién fundado Museo de Zoología.

\section{2. ¿Quién era Douglas?}

Douglas nació el 9 de enero de 1936 en Connecticut, y creció allí. Su padre David Robinson era agente de seguros, y su madre Esther Clark había estudiado artes dramáticas, pero se dedicó a la crianza de sus dos hijos, Douglas y David.

Desde niño se interesó por tener y conocer los animales, pero una fuerte alergia al pelo le fue cerrando sus opciones con los de esa característica, por lo que entre sus mascotas desfilaron lagartijas, tortugas, culebras, tritones, ranas y renacuajos. De las mascotas en terrarios pasó a formalizar sus conocimientos a través de una carrera universitaria por lo que realizó sus estudios en ciencias y biología.

Estudió en Haverford College (Philadelphia) (1953-1955) y en Grinnell College (Iowa) (1956-1958), la maestría la realizó en la Universidad de Michigan (1958-1960), en 1960 fue a la Universidad de Florida y luego se trasladó a la Universidad de Texas A\&M, donde se graduó para su doctorado en 1968 (Chaves y Cornelius, 1991). Participó en proyectos y cursos que le permitieron viajar a la parte tropical de México, Panamá y Costa Rica por lo que estuvo fuera de la universidad por tres años.

Su madre le inculcó el amor por la música clásica, las obras musicales, el cine y el buen rock. Memorizaba y cantaba el guion de obras completas, tocaba varios instrumentos musicales entre ellos el acordeón, el piano y el órgano. Uno de los recuerdos más permanentes que conservo de él es verlo tocando la canción Piano Man, de Billy Joel, en un bar de un remoto pueblo incrustado en el Blue Ridge Mountain. Esa noche hizo cantar a todos los visitantes del bar. También fue amante de la buena comida, le encantaba cocinar platillos poco comunes para los invitados y en su biblioteca tenía excelentes clásicos de la cocina internacional, algunos heredados de su abuela.

Cabe destacar que su primer grupo de interés científico fue el de las salamandras, inspirado por su primer profesor de biología, el famoso herpetólogo Emmett R. Dunn (1894-1956), a quien conoció en Haverford (Savage, 2002). En este grupo hizo su disertación para la tesis de doctorado titulada Phylogenetic Trends of the Salamanders of the Family Plethodontidae as Indicated by Their Cranial Morphology.

Las salamandras motivaron su interés de por vida, al punto de que publicó dos nuevas especies para Costa Rica: Bolitoglossa diminuta Robinson, 1976 y Bolitoglossa gracilis Bolaños, Robinson y Wake, 1987. Además, Bolitoglossa robinsoni Bolaños y Wake 2009, fue bautizada con su apellido.

En el plano personal, al poco tiempo de haber llegado a Costa Rica se casó con Olga Isabel Méndez Arburola, costarricense, estudiante suya en varios cursos y después profesora de biología. Aunque se separaron y no tuvieron hijos, conservaron una buena relación y una larga amistad. 


\section{Douglas como investigador y mentor}

A Douglas le correspondió vivir una época de renacimiento del interés por conocer la biología tropical, para lo que Costa Rica ofrecía una serie de condiciones particulares que constituían, para un amante de retos, una gran oportunidad de realizar sus sueños. De hecho, varios profesores invitados llegaron en esa época a la UCR, así como el regreso de estudiantes costarricenses que se habían graduado en el extranjero. Adicionalmente, organizaciones como el Centro Científico Tropical (CCT), el Centro Agronómico Tropical de Investigación y Enseñanza (CATIE) y la Organización para Estudios Tropicales (OTS) lograron atraer el interés de investigadores de todo el mundo, promoviendo estudios en taxonomía, ecología, comportamiento y otras áreas de la biología que enriquecieron la gran documentación que existe sobre la biodiversidad del país.

En 1970 obtuvo una plaza permanente en la Escuela de Biología de la UCR como catedrático asociado. Docente infatigable, organizó y enseñó los cursos de herpetología, que después se dividieron en un curso de anfibios y otro de reptiles, anatomía comparada, ecología de poblaciones -junto con Gary Stiles y Sergio Salas- algunos seminarios de graduación con temas específicos, y el curso denominado Problemas especiales de biología, en el que se incluyeron cursos de herpetología sistemática, biología de las tortugas marinas y programación aplicable a biología, este último muy novedoso para la época, con el cual nos incorporó en los temas de estadística y cómputo. Nos daba cursos de programación, aprendimos sobre el código binario y a analizar los resultados de los estudios de poblaciones con la ayuda de las computadoras de la UCR; hasta logró tener una terminal perforadora de tarjetas en la Escuela de Biología, lo cual era todo un privilegio en ese momento.

Las giras de los cursos eran verdaderas expediciones. Caminar en pantanos con el agua a la cintura mientras los ojos brillantes de los lagartos iluminaban las orillas, verlo estirar la mano donde uno no veía nada y luego abrirla con una hermosa rana voladora entre los dedos, recolectar terciopelos con gran habilidad y la regañada de: “inútiles!” cuando el temor o precaución nos hacía fallar en una recolección, son escenas que todos recordaremos.

Incontables anécdotas acompañaron a las diferentes generaciones que en 25 años caminaron a oscuras por bosques, ríos y pantanos tratando de alcanzar el paso de las enormes zancadas que lograba con sus largas piernas. Al final regresábamos con un cúmulo de material para la colección del Museo de Zoología que seguía enriqueciendo el conocimiento de los anfibios y reptiles de Costa Rica. Ahí fue director en dos períodos, y estuvo a cargo de la colección de anfibios y reptiles como su curador hasta que se jubiló en 1990.

Su carácter sistemático se refleja en la calidad de la colección del museo, la cual no solo abarca miles de especímenes y sus localidades, sino que estos están debidamente ubicados y preservados con su respectiva etiqueta. Al entregar la colección, debido a su retiro, esta constaba de más de 12000 especímenes debidamente documentados y de una gran variedad de lugares (Savage, 2002).

Una contribución importante como aporte en la documentación de la distribución de las diferentes especies de anfibios y reptiles del país, fue la elaboración de una lista de especies por bio-regiones, para los cursos de Biología tropical de la OTS en 1971 y que posteriormente, en colaboración con varios autores (Savage, 1980) y junto a una clave para la identificación de

Creative Commons (Reconocimiento al autor-No comercial-Compartir igual 4.0 Internacional
(CC BY NC SA 4.0 Internacional)


especies, se distribuyó entre los asistentes a la Reunión LIII (53) de la Asociación Americana de Ictiología y Herpetología, como una publicación de la Revista COPEIA. Es importante acotar que esta reunión se realizó en Costa Rica en 1973, junto con el Dr. Róger Bolaños, la Lic. Mirna López y el Dr. William Bussing, en ella se auspició uno de los contingentes de científicos más prominentes que se han reunido en el país y fue la primera reunión de esta asociación que se realizó fuera de los Estados Unidos.

Es ineludible mencionar que su oficina y biblioteca eran su verdadero santuario. Los grandes clásicos de la literatura en anfibios y reptiles estaban ahí, disponibles para la consulta de todos los que tuvieran interés. No solo los grandes libros, sino también miles de separatas organizadas por autor en varios bloques de archivos.

Cuanto artículo en el tema se daba a conocer en el "Biological abstracts" Douglas lo solicitaba. Tal vez ahora no parezca tan importante ese gran recurso, pero en aquellos años, cuando no existía la Internet, nos tocaba hacer la revisión bibliográfica en esos famosos documentos que estaban publicados en letra diminuta. La búsqueda nos consumía muchas horas en la biblioteca de la UCR; luego de identificar los artículos de interés, había que enviarle una nota por correo al autor y esperar que después de algunos meses el autor se dignara a contestar a un estudiante de una universidad en América Central. Muy diferente era que el Dr. Douglas Robinson lo solicitara o lo tuviera disponible en su biblioteca.

Su vocación docente trascendía lo estrictamente académico, ya que se interesaba que sus estudiantes tuvieran una amplia visión del mundo. Por tanto, promovió que varios de nosotros, lo acompañáramos a reuniones internacionales y a que muy principiantes en el mundo internacional de la ciencia, tuviéramos la oportunidad de exponer ante grandes públicos.

A veces viajábamos por días, en el carro que sus padres nos prestaban, por las carreteras de los Estados Unidos para llegar desde Miami a donde fuera la reunión. Cruzamos montañas y caminos secundarios para visitar sitios muy especiales y en la fecha precisa para encontrar una pequeña laguna para ver la migración de tritones, detenía el carro en cualquier sitio para enseñarnos especies que no teníamos en Costa Rica. Acampamos en parques estatales y nacionales, desde ahí visitábamos los pequeños pueblos montañeses o jugábamos a inventar historias mientras cocinábamos al fuego un sabroso $T$ bone. Durante el camino practicábamos las presentaciones en inglés, nos hacía recitarlas una y otra vez hasta aprendernos la charla, aun así, coleccionamos muchas historias de las cosas que dijimos mal o que nunca nadie entendió de nuestra charla.

Como las reuniones por lo general se realizaban en la primavera, muchas veces correspondió celebrar el día de San Patricio (su santo predilecto) en alguna taberna irlandesa, tenía una serie de paradas obligadas donde buscaba platillos exquisitos, sitios muy particulares que le recordaban su infancia y juventud, lo que nos hacía desviarnos varios kilómetros de las carreteras principales para encontrar el objetivo.

Si bien era muy difícil entender su complejo español, sus murmullos y gestos, por lo que muchos estudiantes no lograban tomar el ritmo de sus lecciones ni la dinámica de su estilo para enseñar, decenas de estudiantes pasamos por sus cursos y logramos graduarnos. Fue tutor de tesis de 12 estudiantes en licenciatura y maestría en temas de herpetología, la mayoría en tortugas 
marinas, motivados por la importancia que adquirió el tema, pero también otros aspectos importantes de la biología y ecología de diversas especies de herpetofauna. En anfibios presentaron tesis Uriel Barrantes y Federico Bolaños. En reptiles José Manuel Mora, Víctor Manuel Martínez, Vilma Castillo y Cruz Márquez, quien estaba en el proceso de redacción cuando Douglas falleció y específicamente en tortugas marinas nos graduamos Rafael Acuña, Fernando Crastz, Mario Alvarado, Adrián Ugalde, Juan Carlos Castro y Ana Cecilia Chaves.

Fue parte del Comité Editorial de la Revista de Biología Tropical por muchos años, y aunque nunca llegó a hablar bien español, su conocimiento de la gramática y su capacidad de identificar el mínimo error tipográfico, ortográfico o gramatical le daba el crédito de excelencia en la revisión.

Para finalizar este apartado, conviene señalar que, por diferentes razones, Douglas no mostró especial interés por publicar en las revistas científicas, debido a esto, sus publicaciones no son, necesariamente, el reflejo de su gran conocimiento y manejo de los múltiples aspectos de la herpetología tropical. Se identificaron 13 publicaciones como autor o coautor en revistas indexadas, en temas relacionados con: la descripción de renacuajos de ranas, aspectos de historia natural de lagartijas, salamandras, tortugas y ranas. También, efectuó estudios de las arribadas de tortugas marinas y sus migraciones, al igual que documentó, con bases científicas, la justificación para que se autorizara la cosecha controlada de los huevos de tortugas, a lo cual se aludirá más adelante.

\section{El científico conservacionista}

Douglas participó en diferentes organizaciones gremiales y de conservación al promover el estudio y conservación de la naturaleza en general, como la Asociación Costarricense para la Conservación de la Naturaleza (ASCONA), el Colegio de Biólogos de Costa Rica y de organizaciones internacionales como American Society of Ichthyologists and Herpetologists, American Society of Zoologists, Biological Society of Washington, Ohio Herpetological Society y Sigma Xi.

Una cuestión que marcó fuertemente el trabajo de Douglas fue el estudio de las tortugas marinas. En 1970 fue informado de que grandes grupos de tortugas anidaban en una estrecha franja de tierra en una playa de la Península de Nicoya, y fue con voluntarios del Cuerpo de Paz a explorar de que se trataba, a partir de ahí se empezó a documentar el fenómeno de las arribadas en Ostional.

En el mismo año, en un conteo aéreo (Richard y Hughes, 1972) tuvieron la oportunidad de dar a conocer la magnitud del fenómeno que ocurría en playa Nancite y en playa Ostional, por lo que se generó gran interés internacional. En 1973, se publicó un artículo en National Geographic (Zahl, 1973) que evidenciaba la diferencia entre las arribadas en ambas playas, sobre todo en que la primera tenía una categoría de protección total por estar ubicada en el Parque Nacional Santa Rosa y en la segunda existía una comunidad que explotaba el recurso, además de animales domésticos y silvestres que excavaban nidos, por lo que se reportaba una situación caótica. Desde un inicio era muy claro que las dos playas no se podían gestionar con la misma regla. Por esa razón, se había propuesto que Ostional tuviera la categoría de refugio, con la posibilidad de darle un manejo distinto. 
Douglas participó en el equipo de trabajo que promovió la creación del Refugio Nacional de Vida Silvestre Ostional en 1982, junto con Guillermo Canessa Mora, Eduardo López Pizarro y la autora, participamos en diversas reuniones con diputados y hasta fuimos atendidos en la casa presidencial por el presidente Rodrigo Carazo Odio, hasta lograr primero el decreto de creación del refugio que cubría 200 metros de la zona marítimo terrestre, desde Punta India hasta el Río Nosara; luego se nos unió la Asociación Cívica de Nosara y se logró la ley con la ampliación del refugio hasta Punta Guiones.

En 1980, junto con su colega Steve Cornelius, quien había venido de voluntario al proyecto años atrás, lograron el apoyo del Fish and Wildlife Service de los Estados Unidos para financiar un estudio de cinco años comparando las arribadas de playa Nancite y playa Ostional. Los informes de este estudio incluían datos sobre los movimientos de la tortuga lora (Lepidochelys olivacea) por el pacífico americano, se analizó la sobrevivencia de los nidos, el impacto de arribadas subsecuentes en nidos colocados previamente, y se detalló la complejidad del fenómeno.

Entre los productos del estudio, está la propuesta para contabilizar las arribadas (Figura 1). Fue necesario un ejército de colaboradores para recoger la información para crear una fórmula que posibilitara contabilizar el tamaño de una arribada. Quienes en su vida han tenido el privilegio de observar este maravilloso fenómeno de la naturaleza, tratarán de organizar en su mente como contabilizar miles de tortugas caminando por la playa para arriba y para abajo, unas ya pusieron, otras van en camino, otras se regresaron sin poner y volverán a salir otra vez, por lo que la primera propuesta implicaba una gran cantidad de probabilidades, constantes y variables que al final permitía estimar cuántas tortugas habían anidado durante los tres o siete días que duraba la arribada, sin sobreestimar el grupo que anidó. Años después la fórmula fue simplificada al conteo de solo las tortugas que efectivamente habían anidado y al evaluar las dos propuestas los resultados fueron comparables (Gates et al., 1996).

Las tortugas marinas significaron un cambio en la perspectiva de su acercamiento a la ciencia, ya que le dio un enfoque integral y que hoy lo enmarcamos en los temas de desarrollo sostenible. Douglas vio en la abundancia del recurso que se presenta en las playas de arribada la posibilidad de un aprovechamiento racional, propuso opciones para que la comunidad de Ostional pudiera aprovechar los huevos de tortuga que por condiciones naturales se iban a perder. Asimismo, los mecanismos de control y administración del recurso, para asegurar que los huevos provenientes de otros sitios de anidación no ingresaran al mercado. 


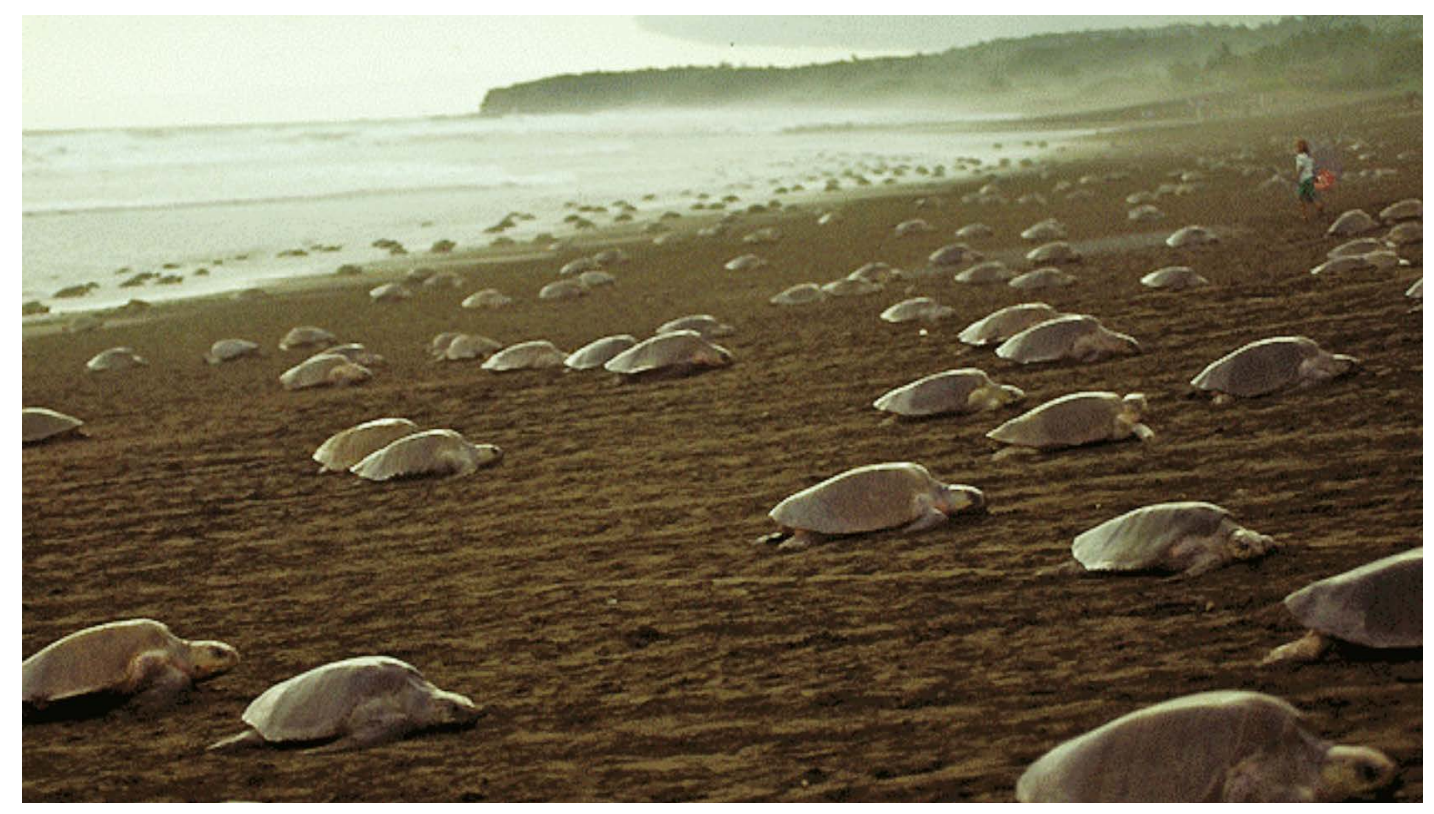

Figura 1. Arribada en Ostional. Fotografía: Akinobu Mochizuki

La situación era muy compleja porque la población de Ostional recolectaba los huevos para vender, mientras que se intentaba estudiar y proteger a las tortugas. No se identificaba el papel que la información podía cumplir en la posibilidad de que se autorizara una recolección regulada. Douglas intentó establecer una estación de investigación, construyó una modesta vivienda que servía para albergar a los voluntarios del proyecto, pero esta fue incendiada.

Por 10 años se trabajó con mucho esfuerzo y recursos limitados, hasta tener la suficiente documentación para lograr incorporar primero en la Ley de Creación del Refugio de Vida Silvestre Tamarindo y luego en la Ley de Fauna Silvestre, un transitorio que permitiera una cosecha regulada de los huevos de tortuga, particularmente en Ostional y la autorización a la Asociación de Desarrollo Específica de Ostional, para comercializar los huevos bajo estrictas normas que permitieran controlar la trazabilidad del origen de los huevos. Se pretendía que al permitir la venta exclusiva de huevos de Ostional a un precio bajo, no fuera buen negocio traer huevos de otras playas que requerían un gran esfuerzo y bajo rendimiento económico.

En 1983 se organizó la primera reunión sobre tortugas marinas del pacífico americano, de la cual surgió la coordinación de proyectos del área en un programa de marcación integrado, del cual Costa Rica era la sede. El proyecto de investigación en tortugas marinas creció a convertirse en un programa que integraba investigación, docencia y acción social. Los estudios de investigación cubrieron otras playas de anidación en el país, se logró la participación de estudiantes costarricenses en los estudios en Tortuguero y se apoyó a las comunidades costeras con la formación de guías locales para llevar a los turistas a observar la anidación sin impactar negativamente en el proceso. En esta tarea acompañé a Douglas hasta que se jubiló y sostuve el programa hasta julio de 1997, cuando por decisión de la Escuela de Biología el programa como tal fue cerrado. 
En 1990 se volvió a construir un edificio para establecer una estación de investigación para el albergue de investigadores, estudiantes y voluntarios, así como, para los cursos de tortugas marinas que diferentes organizaciones solicitaban. La Estación Biológica Ostional fue dedicada a su nombre, en 1996, con la participación de su madre y hermano (Figura 2).

En 1997 se construyó también el Centro para la investigación de las Tortugas Marinas Douglas Robinson en Ostional, el cual sufrió la suerte de la primera estación y fue incendiado el 4 de julio de 1998.

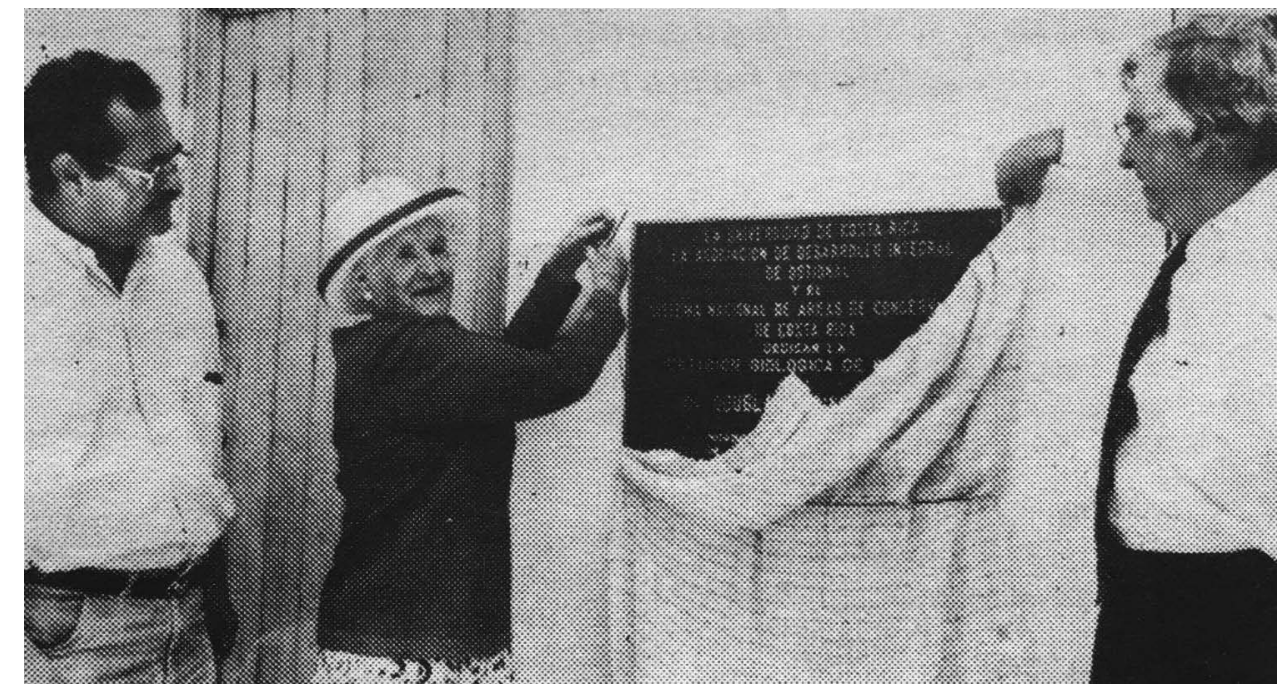

Figura 2. Develación de placa dedicada a Douglas Robinson en la Estación Biológica Ostional, por su madre Esther y su hermano David, en presencia del Dr. Luis E. Garita, por entonces rector de la Universidad de Costa Rica. Fuente: Semanario Universidad (9 de febrero, 1996).

\section{Epílogo}

Como muchas mentes brillantes, Douglas no siempre se sentía cómodo en grandes grupos, tenía una personalidad nerviosa y mostraba tics, era un fumador empedernido, constantemente salía de su oficina para sentarse a la orilla del caño en el parqueo, para fumarse tranquilo un cigarro y entablar una conversación sobre temas diversos con los que por ahí se sentaban para un café o una merienda.

Solía refugiarse en la música y la acompañaba con alcohol. Era frecuente hallarlo en bares de los alrededores de la UCR, en especial el Copas Bar. Para muchos de los que nos graduamos con él, ese bar fue una extensión de la oficina, ahí revisábamos los manuscritos y discutíamos las inconsistencias que encontraba en nuestros textos.

Decidió acogerse a la pensión en forma temprana y se retiró a vivir en una finca en San Isidro de Heredia. Ahí le visitaba los miércoles, le llevaba comida o los insumos para cocinar, a veces se le ocurría que quería cocinar alguno de sus exóticos platillos, creo que en esos tiempos era su comida de la semana, porque de otra manera no sentía apetito. 
Se fue consumiendo poco a poco en el alcoholismo, que al final debilitó su salud, sufrió una caída y estando en el hospital contrajo una neumonía. El 8 de junio de 1991, estando yo en Ostional, llegó personalmente don Daniel Camareno, dueño de la pulpería donde estaba el único teléfono del pueblo, con lágrimas en los ojos, me dio la noticia del fallecimiento de Douglas.

Su temprano fallecimiento conmovió a colegas, exalumnos y amigos, varios obituarios fueron publicados en revistas, noticiarios y semanarios señalando sus aportes y su condición humana, como lo hicieron Julián Monge, Mahmood Sassa, Luko Hilje, Federico Bolaños, Steve Cornelius y quien esto escribe.

Sus restos fueron trasladados por su familia a su tierra natal, aunque algunos amigos manifestaron, posteriormente, que él había hecho arreglos para quedar en Costa Rica, no tuvimos conocimiento de esa información en el momento de su partida. Lo sobreviven sus sobrinos Kimberly y Clark Robinson, hace un par de años recibí una carta de la esposa de Clark donde me contaba que un hijo de ellos estudia biología.

En la edición revisada del libro Biología de las tortugas marinas, Karen Björndall (1995) dedica la edición a Archie Carr, Douglas Robinson y Leo Brongersma de la siguiente forma: "Podemos ver más allá porque estamos en los hombros de gigantes, a estos tres gigantes, con todo respeto, dedico esta edición revisada."

Douglas fue un gigante, pero un gigante con hombros muy amplios, porque dio la base no solo para el estudio de las tortugas, sino también para las salamandras, las ranas, las serpientes, las lagartijas, y toda la herpetofauna, muchos nos pudimos parar sobre sus hombros para ver más allá y seguir su trabajo en la medida en que nos lo permitieron.

\section{Referencias}

Björndall, K. (Ed.). (1995). Biology and Conservation of Sea Turtles. Revised Edition. Washington, EE.UU.: Smithsonian Institution Press. 615 p.

Chaves A. y S. E. Cornelius. (1991). Douglas Robinson (1936-1991): In Memory. Marine Turtle Newsletter 54, 35-36.

Gates, Ch., Valverde, R., Mo, C., Chaves, A., Ballestero, J. y Peskin, J. (1996). Estimating arribada size using a modified instantaneous count. J Agric Biol Environ Stat (JABES), 1(3), 275-287. doi: $10.2307 / 1400519$

Richard, J. D. y Hughes, D. A. (1972). Some Observations of Sea Turtle Nesting Activity in Costa Rica. Marine Biology, 16, 297-309. doi: 10.1007/BF00347753

Savage, J. M. (1980). A Handlist with Preliminary Keys to the Herpetofauna of Costa Rica. UCLA, Los Angeles, EE. UU.: Hancok Foundation.

Savage, J. M. (2002). The Amphibians and Reptiles of Costa Rica. A Herpetofauna between two continents, between two seas. Chicago, EE. UU.: The University of Chicago Press.

Zahl, P. (1973). One strange night on Turtle Beach. National Geographic. pp. 571-580. 